Itinéraires Itinéraires

Littérature, textes, cultures

2009-3 | 2009

Modernités occidentales et extra-occidentales

\title{
Bankim Chandra Chatterji : les ruptures intérieures de la modernité littéraire indienne
}

Claudine Le Blanc

\section{OpenEdition}

Journals

Édition électronique

URL : http://journals.openedition.org/itineraires/498

DOI : 10.4000/itineraires.498

ISSN : 2427-920X

Éditeur

Pléiade

Édition imprimée

Date de publication : 1 novembre 2009

Pagination : 103-119

ISBN : 978-2-296-10115-9

ISSN : 2100-1340

Référence électronique

Claudine Le Blanc, «Bankim Chandra Chatterji : les ruptures intérieures de la modernité littéraire indienne », Itinéraires [En ligne], 2009-3 | 2009, mis en ligne le 11 août 2014, consulté le 20 avril 2019.

URL : http://journals.openedition.org/itineraires/498 ; DOI : 10.4000/itineraires.498

\section{(ब) $(\Theta \Theta$}

Itinéraires est mis à disposition selon les termes de la licence Creative Commons Attribution - Pas d'Utilisation Commerciale - Pas de Modification 4.0 International. 


\title{
Bankim Chandra Chatterji : les ruptures intérieures de la modernité littéraire indienne
}

\begin{abstract}
Known as the first Indian great novelist, Bankim Chandra Chatterji (1838-1894) was both progressist and traditionalist. Analyzing two of his writings which deal with the question of equality between men and women, his essay Samya and his masterpiece KRSNakânter Uil, the present article tries to explore the writer's contradictions, in an attempt to apprehend Indian modernity as a complex phenomenon where past and present, West and East, criticism and mysticism, as soon as set face to face, mingle, the novel being the product as well as the voice of this modernity.
\end{abstract}

Keywords : Bengal, Bankim, essay, novel, equality

Mots clés : Bengale, Bankim, essai, roman, égalité

Contrairement à ce qui s'est produit dans la plupart des cultures extraeuropéennes entrées en contact avec l'Occident, la modernité en Inde n'a pas présenté le visage uni d'une rupture avec la tradition. Dans l'Inde sous domination britannique, l'occidentalisation de la culture et de la société a été dans le même temps conçue comme un ressaisissement de la civilisation indienne : l'expression de « renaissance bengalie » utilisée pour désigner le bouleversement intellectuel des élites à Calcutta lors des premières décennies du XIX ${ }^{\mathrm{e}}$ siècle témoigne de la complexité de la modernisation indienne, sous l'influence d'une Europe qui connait de son côté, avec les travaux des orientalistes et la découverte des grands textes de l'Inde, une "renaissance orientale ${ }^{1} »$. Dans un temps qui conjoint

1. L'expression, reprise en 1950 par Raymond Schwab pour son ouvrage de référence ( $\mathrm{La}$ Renaissance orientale, Paris, Payot), est empruntée à un article homonyme d'Edgar Quinet paru en 1841 dans La Revue des Deux Mondes (n ${ }^{\circ} 28$ ), qui se conclut ainsi : " Le panthéisme de l'Orient, transformé par l'Allemagne, correspond à la renaissance orientale, de même que l'idéalisme de Platon, corrigé par Descartes, a couronné, au XVII siècle, la 
trois époques, Renaissance, Lumières et éveil du nationalisme, l'Inde de la modernité se voit à la fois reconnue dans son ancienneté, saisie par un désir de réforme, et inventée, au sein d'un assujettissement colonial, en tant qu'entité politique propre : le «Raj» des Britanniques. Une telle situation donne lieu à des lignes de fracture en apparence assez claires : d'un côté Rammohan Roy (1772-1833), chantre de Lumières indiennes, grand admirateur de Voltaire dont il donnait Le Siècle de Louis XIV à lire à ses élèves, décrit par Tagore comme «le seul homme de son temps qui ait complètement compris la signification de la modernité »; de l'autre une orthodoxie qui, à partir d'un semblable diagnostic de décadence, œuvre pour une rénovation de l'intérieur, et rejette toute innovation, qu'il s'agisse de la Satee Regulation (1829) contre la pratique de la crémation des veuves ou du Calcutta Medical College (1835). L'opposition, qui reconduit la définition de la modernité comme rupture, confirme aussi l'idée selon laquelle la modernité est d'essence européenne ${ }^{2}$, désirée par les uns, refusée par les autres. Les démarcations, pourtant, sont plus tortueuses, et passent au cœur des êtres, des pensées, des sensibilités : parfois a posteriori, comme pour Rammohan Roy dont l'héritage se partage entre rationalistes, tel Ishwar Chandra Vidyasagar (1820-1891), qui se placent sous l'influence de Hume, Mill et des Utilitarians anglais, et de plus inattendus mystiques qui tendent à faire du Brâhmo Samâj, fondé par Rammohan, un courant religieux réformateur parmi d'autres, transmettant l'enseignement d'un saint. Avec l'émergence de la question nationale, les clivages par ailleurs viennent à se brouiller. La Bengal British India Society fondée en 1851 a pour président le champion de l'orthodoxie brahmanique, Radhakanta Deb (1784-1867), et pour premier secrétaire Debendranath Tagore - le père de Rabindranath - qui est à la tête du Brâhmo Samâj : les anciens adversaires s'unissent dans un mouvement de renouveau (en anglais revivalism) qui entretient l'idée que les misères et les injustices régnant en Inde sont dues aux envahisseurs étrangers, et particulièrement aux musulmans. " $[\mathrm{T}]$ he cause of the state of seclusion and imprisonment in which the females of this land are preserved may be traced to the tyranny of the Mohammedan Emperors ${ }^{3}$ ", écrit Maheshchandra Deb, ancien élève de Henry Derozio (1809-1831) qui fut démis de ses fonctions au Hindu College par Radhakanta Deb pour un enseignement trop hétérodoxe. Dans la seconde moitié du XIX ${ }^{e}$ siècle, les antagonismes au sein de l'élite hindoue anglicisée se révèlent ainsi mouvants selon les questions abordées. On attribue parfois ces phénomènes de dissolution des oppositions à une tendance propre de l'hindouisme au

renaissance grecque et latine. »

2. «La modernité. Inutile d'ajouter : occidentale. La modernité est européenne. [...] La modernité part de l'Occident, et revient à l'Occident. Elle est l'Occident », Henri Meschonnic, Modernité modernité, Paris, Gallimard, 1994 [1988], coll. « Folio », p. 27.

3. Cité par Gautam Chattopadhyay, Awakening in Bengal in Early Nineteenth Century, Calcutta, Progressive Publishers, 1965, p. 94. 
syncrétisme, à l'agglomération ${ }^{4}$. Mais il faudrait sans doute se demander s'il ne s'agit pas plutôt de la forme que prend la modernité en Inde, qui ne serait donc pas un simple emprunt à l'Occident, mais rejouerait sur la scène indienne l'ensemble du processus moderne comme émergence d'un présent et d'un sujet. Dans le domaine de la littérature, la modernité se manifeste par l'apparition de genres tels que l'essai et le roman, mais ces deux modes d'écriture peuvent en venir à jouer l'un contre l'autre dans un même travail du sujet: exemplaires de ces tensions qui ne sont pas des ruptures, ou qui sont des ruptures intérieures, apparaissent ce qu'on peut appeler les deux modernités du bengali Bankim Chandra Chatterji (1838-1894), premier grand romancier indien, successivement progressiste et conservateur, simultanément progressiste dans un essai $\left(\right.$ Sâmya $\left.{ }^{5}\right)$ et conservateur dans un roman (KRSNakânter Uil).

\section{Bankim Chandra Chatterji et l'avènement de la modernité en Inde}

Né dans une famille de brahmanes du Bengale rural, Bankim Chandra Chatterji appartient à la première génération d'Indiens ayant reçu l'éducation anglaise imposée par la circulaire Macaulay de 1835 : entre onze et dix-huit ans, il étudie au Hugli College et découvre la littérature et la pensée anglaises, qu'il maîtrise de façon remarquable. C'est dans un second temps, à quinze ans, que le jeune homme éprouve le besoin d'apprendre le sanskrit et se met à fréquenter une école traditionnelle, sans doute parce qu'il partage l'amour que sa famille porte à la littérature bengalie et ressent la nécessité d'en connaître les sources. Dans cette triple formation qui caractérise les créateurs de son temps, en un parcours à chaque fois singulier (Ishvar Chandra Vidyasagar apprit l'anglais après des études traditionnelles en sanskrit, Michael Madhusudan Dutt au contraire se tourna tardivement vers le bengali et le sanskrit), apparaissent déjà les trois - et non deux - éléments de la modernité indienne : la tradition villageoise en sanskrit, la culture scolaire anglaise, et ce qui tient en Inde à la fois de l'ancien et du moderne, la littérature des langues vernaculaires.

Étudiant en droit à Calcutta au Presidency College puis à la toute nouvelle université, Bankim ne se rapproche ni du Brâhmo Samâj, ni des Deroziens : il semble se tenir en retrait de tous les mouvements sociaux et religieux qui agitent la ville, mais lit beaucoup, ce qu'il continua à

4. Voir, par exemple, Renaissance and Reaction in Nineteenth Century Bengal: Bankim Chandra Chattopadhyay: An English translation of the Bengali essay Samya written by Bankim Chandra Chattopadhyay, the father of Indian Nationalism, traduction et introduction de M. K. Haldar, Calcutta, Minerva Associates, 1977, p. 86.

5. Les titres bengalis et sanskrits sont translittérés selon les conventions suivantes : l'accent circonflexe note une voyelle longue, la majuscule un son rétroflexe, "sh » enfin représente le s palatal. Afin d'alléger la lecture, on a adopté pour les autres termes (noms de lieux, de personnages, etc.) une transcription simplifiée. 
faire tout au long de sa carrière de Deputy magistrate. En 1864, il publie en feuilleton dans Indian Field un roman en anglais, Rajmohan's Wife, qui reste inachevé. La même année, il se lance dans la rédaction de son premier roman bengali, Durgheshnandinî, inaugurant une série de romans historiques ou sociaux en bengali qui fait de lui le père du roman indien. Le tournant est décisif, mais n'est pas dénué d'ambiguïtés : lorsque Bankim rédige Rajmohan's Wife, Michael Madhusudan Dutt a déjà fait le choix du bengali; en renonçant à l'écriture en anglais, Bankim semble en même temps abandonner le thème du malheur conjugal féminin auquel Rajmohan's Wife est consacré, puisque Durgheshnandinî s'inscrit dans le genre du romance. Les années qui suivent voient cependant se multiplier, parallèlement aux romans, des essais en anglais et en bengali, pour la plupart publiés à partir de 1872 dans sa revue Bangadarshan, où paraissent aussi, en feuilleton, les romans. Tout se passe comme si l'essai permettait à Bankim d'engager un débat avec la modernité anglaise, où l'auteur fait bien souvent preuve d'une ironie mordante qui prend acte de la supériorité britannique tout en en contestant la pérennité par la possibilité offerte à l'Inde, du même coup, d'en subir l'influence positive pour renouer avec le meilleur d'ellemême. En 1873, par exemple, The Study of Hindu Philosophy paru dans Mookerji 's Magazine brosse un tableau extrêmement critique de la méthode philosophique indienne :

An intense theological spirit rarely leads to anything but the deductive method, and the Hindu method was almost solely and purely deductive. Observation and Experiment were considered beneath the dignity of philosophy and science. Nor is even deduction as rule pushed on to its legitimate consequences. First principles are assumed on no grounds, and with the most perfect weapons of deductive logic at his command, the Hindu thinker contents himself with the most fanciful inferences ${ }^{6}$.

Bankim épouse ainsi un point de vue occidental sur la philosophie indienne, au point où sa position a pu, aux yeux de certains, apparaître proche de celle de Hegel dont il n'était pas, semble-t-il, familier ${ }^{7}$. Mais sans connaître les Leçons sur la philosophie de l'histoire et la Philosophie de la religion, Bankim connaît la principale source de Hegel, l'History of British India de James Mill (1817) et se montre apte à développer une critique de l'extérieur. Faut-il parler d'aliénation en reconnaissant en Bankim un produit de la politique éducative préconisée par Macaulay qui visait à créer « a class of persons Indian in blood and colour, but English in tastes, in opinions, in morals and in intellect »? Rien n'est moins sûr. Dans un second temps, caractéristique du mouvement de sa pensée, Bankim s'emploie à repérer

6. Bankim Rachanabali, J. C. Bagal (éd.), Calcutta, Sahitya Samsad, 1953-1969, III, p. 149.

7. Voir Sisir Kumar Das, The Artist in Chains. The Life of Bankimchandra Chatterji, New Delhi, New Statesman Publishing Company, 1984, p. 60. 
des similitudes entre certains points des systèmes philosophiques indiens (darshana) et la philosophie occidentale : ainsi la notion de loi absolument souveraine, qui confère selon lui à l'Europe moderne une supériorité incontestée sur l'Europe passée et les pays extra-européens présents, se trouve, dit-il, dans les systèmes Sâmkhya et Nyâya ${ }^{8}$.

Une semblable structure à double détente, qui élève la modernité occidentale pour lui égaler, en définitive, une Inde éternelle, se retrouve chez Bankim quand il s'agit des questions de société, celles-là même qui nourrissent ses romans dits sociaux. Quand Vidyasagar, en 1855-1856, multiplie les manifestations en faveur du remariage des veuves et obtient sa légalisation, Bankim, sans adopter la position radicalement conservatrice de Radhakantha Deb, s'oppose au Widow Remarriage Act. Dans un essai paru plus de vingt ans plus tard, Bankim reprend ce point, dans le cadre général d'une réflexion sur l'égalité qui constitue une sorte de synthèse exemplaire de sa modernité.

\section{Sâmya : l'essai sur l'égalité}

Sâmya $a^{9}$ («Égalité ») est a priori remarquable pour la dénonciation qu'il propose du sort réservé aux femmes en Inde : s'y fait entendre le Bankim le plus anglicisé, pétri de références philosophiques occidentales. Mais l'histoire du texte, publié en 1879, révèle un positionnement plus complexe : Sâmya rassemble un long essai rédigé entre 1872 et 1873 sur la condition des paysans au Bengale, Bangadesher Krishak, et deux essais ultérieurs, consacrés au concept d'égalité. Par la suite, Bankim renia Sâmya qu'il retira de la circulation, mais il fit réimprimer avec quelques modifications l'essai sur la paysannerie et l'inclut dans sa collection d'essais Bibidha Prabandha, parue en deux tomes, en 1887 et 1892. Le point de départ (et d'arrivée) est donc paysan; il n'est pas neuf, d'autres l'ont abordé avant ou en même temps que Bankim, tel Ramesh Chandra Dutt dans le Bengal Magazine en 1873 et, de ce point de vue, Bankim se fait avant tout la chambre d'échos de combats contemporains autour du Permanent Settlement Act de 1793 en particulier qui, réglementant et fixant le lien des paysans au zamindar ${ }^{10}$, en alourdissait l'assujettissement. Mais il s'empare de la question avec un emportement qui, d'une certaine façon, se communique au dernier chapitre, lequel traite de l'inégalité entre les hommes et les femmes.

8. Bankim Rachanabali, op. cit., p. 148.

9. Traduit en anglais par M. K. Haldar dans Renaissance and Reaction in Nineteenth Century Bengal : Bankim Chandra, op. cit., p. 149-303 [désormais désigné par la lettre S]. 10. D'origine persane, le terme désigne les responsables du maintien de l'ordre et de la collecte des taxes dues par les cultivateurs dans le système fiscal, administratif et social en usage dans les régions sous domination de l'Empire moghol à partir du XVI siècle. Les zamindar, dont la charge était devenue héréditaire, furent assimilés à des propriétaires terriens par les Britanniques au Bengale. 
S'adressant au lecteur moyen de Bangadarshan, Bankim dans son essai propose une analyse qui se veut accessible; il ne s'embarrasse ni de nuances ni d'originalité. Reprenant l'analyse de Rousseau dans le Discours sur l'origine de l'inégalité, il distingue deux types d'inégalité, une diversité de conditions naturelles d'une part, des différences de convention, non naturelles, d'autre part, qui s'ajoutent aux premières. Dans le cadre des inégalités naturelles, Bankim fait entrer non seulement la force ou la beauté, comme Rousseau, mais aussi les conséquences de celles-ci, qui sont déjà des inégalités sociales, telles que la fortune matrimoniale (une femme belle épouse un zamindar quand une femme moins belle devra vivre en coupant du jute) ou la considération (un homme intelligent est respecté, un homme qui l'est moins est méprisé). L'inégalité naturelle pour Bankim est conçue comme une diversité (il emploie le terme baisâmya et non l'antonyme construit avec le préfixe privatif, asâmya), or la diversité est une loi du monde. De ce point de vue, les inégalités sociales sont aussi des inégalités naturelles : au moment même où il pose la distinction entre les deux inégalités, d'un ton vif, sarcastique, et dans un registre imagé, Bankim tend à la brouiller. Il y a en Inde, expose-t-il, deux types d'hommes importants : le riche, qui peut bien avoir accumulé des biens par le moyen $\mathrm{du}$ vol - et il propose une petite vignette d'un riche et odieux Ram -, et le brahmane, illustré cette fois par un Gopal Thakur qui, tout désargenté qu'il soit, n'en reste pas moins un grand homme dont un shûdra - un homme de la caste des serviteurs - doit toucher les pieds. Contrairement à ce que la rhétorique laissait supposer, les deux types appartiennent bien au même cas des inégalités de convention ${ }^{11}$.

L'enjeu se trouve par là déplacé du qualitatif au quantitatif : ce n'est pas tant la nature des inégalités que les effets pervers de leur excès qui posent problème. Or, si la diversité existe nécessairement partout, en Inde les différences sociales sont excessives, et cet excès empêche le progrès social. D'autres pays ont pris des mesures pour minimiser ces inégalités : Rome par une intelligente politique d'intégration des étrangers, la France ou les États-Unis par la «chirurgie»- Bankim évoque la « récente» guerre de Sécession. Mais le plus souvent ce sont les mots qui ont permis l'établissement de l'égalité, et Bankim cite alors trois grands hommes qui, en des temps de crise, ont prêché avec succès l'égalité des hommes : Bouddha dans l'Inde védique écrasée par le privilège des brahmanes monopolisant le savoir à leur profit ; Jésus-Christ dans l'Empire romain divisé entre maîtres et esclaves; le « troisième avatar », apparu dans la France du XVIII ${ }^{e}$ siècle en proie à de criantes inégalités sociales, étant Rousseau, auquel tout le chapitre suivant se consacre.

11. Bankim signale alors en passant qu'il existe aussi une différence de convention entre les indigènes et les étrangers, tout particulièrement les Anglais, mais il ajoute aussitôt qu'il préfère ne pas engager de débat sur ce point. 
Le raisonnement de Bankim semble articuler deux plans contradictoires : tout en s'inspirant de Rousseau et en faisant d'ailleurs de celuici un des prophètes ayant révélé l'égalité essentielle des hommes, il pose, cette fois contrairement à Rousseau, que l'inégalité est une loi de la nature, sous la forme de la diversité, si bien qu'il en arrive à faire de l'égalité une pure création des sociétés. Il y a là pour une part une compréhension insuffisante de l'analyse de Rousseau - au lieu de l'état de nature, Bankim brosse un tableau idyllique de l'Inde historique bouddhique -, mais aussi une fascinante tension intérieure entre un désir de justice sociale - et Bankim pointe bien ce qui en Inde est le plus inégalitaire, à savoir le statut, c'est-à-dire la caste - et l'impossibilité de supporter toutes les implications d'une égalité de nature.

Le deuxième chapitre, qui traite de Rousseau, devient ainsi un tableau à charge : Rousseau est le père spirituel des Français mais, à la différence du Bouddha et du Christ, il a prêché une vérité non pure, dont la force reposait sur la " magie de ses mots », et qui mêlait vérités salutaires et erreurs préjudiciables. La thèse selon laquelle l'égalité est une loi naturelle est une parole dangereuse, déclare Bankim après avoir cité en les abrégeant les célèbres premières lignes de la seconde partie du Discours (« Le premier qui ayant enclos un terrain...»). Opérant implicitement une distinction entre valeur intrinsèque et valeur pragmatique d'une proposition, Bankim ne dit cependant pas que l'égalité naturelle est une erreur, mais qu'elle entraîne des conséquences néfastes. C'est donc l'efficacité de la thèse dans le réel qui la rend contestable. Le Contrat social est ainsi à la source de la Révolution française : «Le jour où le Contrat social a été publié, ce jourlà le sceptre dans les mains du roi de France a été brisé ${ }^{12}$.» Et, avec la Révolution française, l'ordre des choses a été définitivement changé : les méprises de Rousseau, dit-il, devinrent les fondations d'un bien permanent pour l'humanité, parce que ces méprises étaient prêchées sur la base d'une vérité, à savoir l'égalité. Mais quelles sont donc ces erreurs de Rousseau? Sont-ce les conséquences qu'il tire de la doctrine de l'égalité naturelle? Le propos de Bankim reste opaque, d'autant que dans la dernière partie du chapitre deux, il s'emploie à détailler, sans jugement négatif semble-til, les «fruits » du rousseauisme au XIX ${ }^{\mathrm{e}}$ siècle : les «communismes » de Louis Blanc, Saint-Simon, Charles Fourier, mais aussi la philosophie de John Stuart Mill en matière d'héritage et de droits des femmes. Là, Bankim redevient d'un progressisme qui peut être qualifié de radical : il énonce les théories de Mill en les reprenant à son compte sans réserve, faisant droit à leur justesse qui semble en rendre la réalisation nécessaire - comme plus haut au sujet de Rousseau, la vérité est douée d'efficace. Les lois sur la succession sont partout injustes, la proposition de Mill de limiter l'héritage des enfants à leurs stricts besoins peut faire sourire, dit-il, mais un jour elle

12. S, p. 162; la traduction française est de l'auteur. 
s'appliquera dans le monde entier. De même, l'idée que les femmes ne sont pas aptes au savoir ou à des activités professionnelles n'est qu'un préjugé populaire tenace dont l'Europe s'est dépouillée avec profit, mais il faudra sans doute des années pour qu'il en aille de même en Inde.

"Sâmya is full of errors ${ }^{13}$ ", a dit Bankim lorsqu'il a renié son essai. Mais quelles sont, ici aussi, ces erreurs? S'agit-il d'une mauvaise appréciation de Rousseau, de Mill, ou de la philosophie de l'égalité tout entière? Celle-ci, telle que Bankim la promeut, est récapitulée de la façon suivante à la fin du chapitre : les hommes sont égaux, mais cela ne signifie pas qu'il n'y ait pas des différences naturelles entre eux, qui entraînent des différences sociales. Ce sont les différences sociales qui ne sont pas le résultat de différences naturelles qui sont injustes et néfastes et qu'il convient de corriger : le statut et la fortune résultant de la naissance, mais aussi du sexe - conçu donc abstraction faite de toute différence naturelle - soit les deux points de John Stuart Mill, et les deux objets des trois derniers chapitres. On en revient à une analyse en termes qualitatifs, qui introduit la mise en fiction du chapitre trois : certaines situations précises sont scandaleuses. Il en va ainsi de la misère de la paysannerie bengalie, incarnée par le personnage de Paran Mandal, qui se trouve emporté dans la spirale de l'endettement à la suite des exactions des percepteurs et des usuriers ainsi que des cadeaux dus au zamindar à diverses occasions, et qui ne peut finir qu'en prison ou en exil.

Le trait est plein de vivacité et non dénué de causticité : le personnage est pris à la gorge sous les yeux du lecteur. La conclusion néanmoins opère un pas en retrait : il ne s'agit en aucun cas de critiquer tous les zamindar, ou s'il faut les critiquer tous, ce sera dans la mesure où ils laissent les pires d'entre eux agir de façon inique. La confiance dans une régulation communautaire plus que dans la loi ne laisse pas de surprendre, d'autant que le chapitre suivant, qui analyse les origines de cet état de la paysannerie en Inde l'attribue à un figement pervers d'un fonctionnement social en soi souhaitable, qui impose d'épargner le travail physique à certains afin qu'ils puissent se consacrer à la connaissance qui est au fondement du progrès de la civilisation. Mais en Inde, en raison des ressources du pays, chaud et fertile, qui ont permis une accumulation rapide de richesse, un accroissement de la population et une certaine apathie renforcée par le nihilisme de la philosophie, la société a été divisée d'emblée et durablement en deux sections où celle qui ne travaille pas accapare tout le profit et dégénère, tandis que le sort de l'autre va s'aggravant au fil des ans. C'est cet état des choses qui s'est mis à agir comme un poison, "le poison de la discrimination ${ }^{14} »$, principale cause du retard du peuple indien. Nourri des analyses économiques d'Adam Smith, H.T. Buckle et des utilitaristes,

13. Cité par Sisir Kumar Das, op. cit, p. 90.

14. S, p. 188. 
le propos est sévère (même si, à sa faveur, Bankim pose en leitmotiv l'exceptionnelle fécondité de la terre indienne).

Le dernier chapitre, consacré aux femmes, prolonge le réquisitoire. Bankim commence par défaire l'argument selon lequel la faiblesse des femmes justifierait leur domination, en menant un parallèle avec la situation coloniale : la faiblesse des Bengalis légitime-t-elle leur asservissement par les Anglais? La différence des forces est aussi un effet de la différence des droits, rappelle-t-il, renvoyant à Subjection of Women de J. S. Mill. C'est ici l'accomplissement de la première modernité de Bankim, abandonnant sa lecture critique de Rousseau, épousant complètement la philosophie de l'égalité de l'Europe et la retournant contre celle-ci, mais aussi bien et plus encore, contre l'Inde. L'assujettissement des femmes existe partout, écritil, mais en Inde, il est encore plus grand: "Notre pays est un pays de sujétion : les graines de toutes les sortes de sujétion sont semées ici; elles trouvent un sol fertile et poussent particulièrement bien ${ }^{15}$. » Plus nettement encore qu'au sujet des paysans, Bankim pointe quelque chose de pervers dans cette Inde si fertile où fleurit l'inégalité. Son propos est d'un rigoureux progressisme, même si, de nouveau, le caractère d'exception qu'il accorde à l'Inde dans le comble de l'inégalité lui confère une irréductible spécificité susceptible de se retourner. Bankim poursuit en passant en revue les cinq lieux où les femmes sont victimes de discriminations: elles n'ont pas accès à l'éducation, ne peuvent se remarier, vivent recluses dans la maison, doivent supporter la polygamie sans pouvoir jouir d'un système équivalent, n'ont pas le droit d'hériter. La question du remariage, ou de la polygamie, montre bien sur quelle base Bankim raisonne : il ne s'agit pas d'évaluer des pratiques en soi mais, en refusant tout raisonnement à partir d'une nature féminine spécifique, de revendiquer dans tous les domaines une égalité effective des droits et des restrictions entre les hommes et les femmes. Si la femme non chaste doit être privée du droit à hériter de son père, il doit en aller de même pour un homme libertin ou adultère. Bankim, qui ne manque pas de dénoncer l'hiatus entre la théorie des progressistes, disciples de Vidyasagar ou adeptes du Brâhmo Samâj, et leur pratique, se fait donc pour finir le partisan d'une égalité des droits sans restriction aucune.

De la diversité naturelle des conditions à l'égalité des droits, le chemin n'est pas sans cohérence, mais on a vu par quels nœuds, par quels décrochages le propos de Bankim se construisait. Hétérogène dans sa composition, Sâmya est resté désarticulé, non lissé, sans doute parce qu'il était impossible de le faire, si bien que s'y lisent à la fois l'accomplissement de la modernité occidentalisante de Bankim, et les points d'achoppement cristallisant ce qu'on appellera sa modernité indienne. 


\section{KRSNakânter Uil : le roman de la destinée}

Le contrepoint antiprogressiste que constitue à première vue l'œuvre romanesque de Bankim pendant la même période est donc à considérer au regard de la complexité de l'essai. Le roman de ce point de vue recompose les accents et, fidèle à sa vocation critique, dramatise les tensions de la pensée en les déployant dans un temps diégétique. Entre 1873, date du Bangadesher Krishak et 1879, date de Sâmya, quatre romans sociaux reprennent en les mettant en scène les enjeux de l'égalité : BiSabRkSa (L'Arbre à poison, 1873), Chandrashekhar (paru en feuilleton en 1874 dans Bangadarshan, 1875 en volume), Rajanî (1873-1874 en feuilleton, 1877 en volume), KRSNakânter Uil / Le Testament de Krishnokanto (publié en feuilleton en 1875-1877, en volume en 1878). Ce sont autant de variations sur une même thématique : l'amour entre un homme marié et une femme, le plus souvent une jeune veuve, et le fruit catastrophique que porte cet arbre vénéneux. Le titre du premier roman indique bien en effet la condamnation véhiculée par la fiction : dans une optique toute différente du chapitre cinq de Sâmya, Bankim ne se cantonne pas à la mise en regard systématique de la condition des hommes et des femmes; il aborde le sens et la valeur du mariage, de la chasteté, c'est-à-dire qu'il noue la problématique de l'égalité des droits aux effets concrets de sa mise en œuvre. Or, sur cet arrière-plan, celle-ci, comme l'égalité de Rousseau, perd une bonne part de sa légitimité par les conséquences qu'elle entraîne. S'il est accessoire, peut-être, comme l'avancent certains critiques ${ }^{16}$, que la femme du couple adultère soit veuve, il n'est certes pas anodin que l'homme dont elle s'éprend soit marié. En ce qui concerne les droits des femmes, quoi qu'il en soit, c'est bien le roman qui assure le pas de côté effectué dans l'essai à propos de l'égalité théorique.

KRSNakânter Uil, le dernier des quatre romans, traduit en français sous le titre Le Testament de Krishnokanto ${ }^{17}$ et quasi contemporain de Sâmya, est généralement considéré comme le chef-d'œuvre de Bankim, à la fois singulier - c'est le seul roman exempt de tout surnaturel - et exemplaire de la technique comme de la pensée controversée de l'auteur. Il orchestre plusieurs des débats de la modernité auxquels Bankim s'est intéressé : le veuvage des femmes, la fortune des zamindar, le droit de la succession, qui lui donne son titre - c'est le terme anglais, will, qui est utilisé. Le point de départ de l'intrigue est un problème de transmission : deux frères d'une famille de zamindar ont exploité ensemble une propriété qui leur a rapporté une fortune immense, mais l'un est mort avant d'avoir pu légaliser par un acte notarié le droit de son fils Gobindolal à hériter. Krishnokanto, le survivant, n'a aucune intention de spolier son neveu, mais il n'en demeure

16. Voir Sisir Kumar Das, op. cit., p. 67.

17. Traduit du bengali, préfacé et annoté par Nandadulal Dé, Gallimard/Unesco, coll. « Connaissance de l'Orient », 1973 [désormais désigné par KU]. 
pas moins que selon le système d'héritage hindou que l'Indian Succession Act de 1865 laisse inchangé puisqu'il ne s'applique pas aux populations hindoues et musulmanes, le testateur est seul maitre de ses dispositions et peut les changer à tout moment ${ }^{18}$. L'intrigue se noue à partir de là : le fils aîné de Krishnokanto, furieux de voir Gobindolal hériter de la moitié de la fortune quand lui-même ne doit en recevoir que trois seizièmes, tente de faire modifier la teneur du testament. Il commence par exercer un chantage sur son père en menaçant d'épouser une veuve; son père le déshéritant alors quasi complètement, il fait substituer un faux testament au vrai par une jeune veuve du voisinage d'une grande beauté, Rohini, à qui il promet le mariage. Peu après, celle-ci, saisie de remords, entreprend d'effectuer l'opération inverse, mais elle est alors surprise par Krishnokanto. Averti par son épouse, Bhromor, de la situation de leur voisine, Gobindolal intervient pour la sauver; cependant Rohini, tombée amoureuse de lui, tente de se noyer. De son côté, Gobindolal n'est pas insensible au charme de la jeune femme mais lutte contre ses sentiments. À la suite d'une rumeur qui circule dans le village, Bhromor croit que Gobindolal est parti avec Rohini alors qu'il s'est absenté pour fuir celle-ci; se refusant à attendre son retour, elle se rend chez son père. Averti également de la rumeur, Krishnokanto modifie une fois encore son testament, substituant le nom de Bhromor à celui de Gobindolal. Krishnokanto meurt, Gobindolal ne parvint pas à oublier Rohini et quitte la demeure familiale. Comme Bhromor dépérit, son père entreprend de rechercher Gobindolal; en corrompant le postier, il repère Gobindolal et Rohini qui vivent incognito dans un village éloigné et leur envoie un émissaire sous un faux prétexte. Entendant le nom de sa femme, Gobindolal se met à pleurer, tandis que Rohini, cédant au désir d'avoir des nouvelles de son village, ou de séduire le jeune homme - le narrateur ne tranche pas -, lui fixe un rendez-vous que Gobindolal, dûment informé par celui-ci, surprend. Il abat Rohini d'un coup de revolver avant de s'enfuir. Arrêté au bout de quelques années d'errance, il est traduit en justice mais son beau-père parvient à corrompre les témoins. Acquitté, Gobindolal disparaît de nouveau mais, à bout de ressources, il demande par lettre à sa femme de lui donner asile. Celle-ci lui répond sèchement que la propriété est à lui, mais elle meurt bientôt et Gobindolal, prévenu par son beau-père, reçoit son dernier souffle. Consumé de souffrance, Gobindolal se réfugie dans une vie d'ermite.

Quelle est la cause d'un tel drame? Cette question, que le narrateur lui-même pose au long de son récit et qui en constitue le sens, n'est peutêtre pas aussi aisée à trancher qu'il peut le sembler. Le lecteur de Sâmya reconnaît dans le roman deux états de fait que Bankim, à la suite de John Stuart Mill, dénonçait : la discrimination vis-à-vis des femmes d'une part (le fils de Krishnokanto, veuf, laisse libre cours à sa cupidité en toute

18. Un débat s'engage toutefois par la suite sur la validité des dispositions de Krishnokanto, dans la mesure où il ne saurait disposer de l'héritage de son frère, voir KU, p. 128. 
impunité ${ }^{19}$; Rohini, veuve, est punie de mort pour ne pas avoir renoncé à vivre); le désordre social provoqué par le principe de l'héritage d'autre part, que Bankim avait personnellement expérimenté pour avoir été écarté de la succession de son père en $1865^{20}$. Mais rien dans le roman ne permet de penser que ces maux sont à la source du drame; au contraire, les gestes modernes que sont le mariage avec une veuve ${ }^{21}$, ou la protestation d'une épouse - car Bhromor elle-même se montre indocile en n'étant pas présente pour accueillir son mari - précipitent la catastrophe. Aussi l'impression du lecteur est-elle bien celle d'une critique de la modernité, et d'un éloge implicite de la tradition. Mais pourquoi la tradition est-elle bonne? Parce qu'elle protège l'homme contre lui-même, son désir, son insatisfaction. Comme le protagoniste de BiSabRkSa, Gobindolal est tourmenté par le désir d'une autre femme, Rohini quand il est auprès de Bhromor, Bhromor quand il est avec Rohini. Là est le poison :

Gobindolal avait aimé deux femmes, Bhromor et Rohini. Toutes les deux étaient mortes. L'insatiable besoin de beauté qu'il portait en lui pendant sa jeunesse l'avait poussé vers Rohini et il avait abandonné Bhromor pour elle. Mais à peine l'avait-il conquise qu'il s'était rendu compte que cette Rohini ne valait pas Bhromor, que la soif de beauté n'était pas l'amour, que la jouissance n'était pas le bonheur, c'était le poison fatal provenant de la respiration de Bashouki écrasé par le frottement de Mondar, et non le nectar s'écoulant du pot de Dhonontori. Il avait compris qu'à force de baratter ce cœur de l'océan à coups répétés, il en avait fait sortir un poison mortel auquel il ne pouvait échapper, qu'il était condamné à boire, et qu'il but comme Nilkontho ${ }^{22}$.

La référence mythologique fait pencher la balance en faveur de Bhromor : de la même façon que lors du barattage de l'océan de lait que les dieux accomplirent pour récupérer le nectar d'immortalité englouti par le déluge, apparurent ensemble l'ambroisie et le poison - secrété par le serpent Vasuki, utilisé comme corde -, de la même façon dans l'histoire de Gobindolal il y eut la paradisiaque Bhromor et la vénéneuse Rohini. La fin du roman était donc inéluctable, et là réside sa signification, comme l'expose une intervention de l'auteur quelques lignes plus loin:

19. « Je suis une voleuse, et toi tu es un saint! Qui est-ce qui m'avait dit de voler? [...] Si tu étais une femme, c'est à coups de balai que je t'aurais chassé aujourd'hui. Mais toi, tu es un homme! », crie Rohini au fils de Krishnokanto qui vient de lui déclarer qu'une femme qui a volé ne pourra jamais être sa femme, KU, p. 50-51.

20. Les raisons en restent obscures. Voir Sisir Kumar Das, op. cit., p. 110.

21. «Tu sais, Rohini, le mariage de veuves est conforme aux Écritures », dit le fils aîné de Krishnokanto à Rohini qui lui répond : «C'est ce qu'on dit maintenant. », KU, p. 44.

22. KU, p. 192-193. Le traducteur a choisi pour les noms propres une graphie respectant la prononciation bengalie. Les noms sanskrits sont respectivement Vasuki, Mandara, Dhanavantari, Nilakantha. 
Bhromor était en lui [...] c'est cette présence de Bhromor dans le cœur de Gobindolal qui entraîna la mort si rapide de Rohini, restée en dehors de lui-même. Si quelqu'un ne l'a pas compris, c'est en vain que j'ai écrit cette histoire.

Bien moins belle que Rohini, Bhromor « le petit bourdon noir » est présente dans le cœur de Gobindolal parce qu'elle est son épouse, et que la femme révèle dans le mariage cette nature féminine spécifique que l'auteur de Sâmya refusait d'envisager : « Les femmes sont toute indulgence, toute bonté, tout amour : elles sont la plus parfaite réalisation de Dieu; la femme est l'ombre de Dieu; l'homme n'est que sa création ${ }^{23}$. » Et Bankim, après avoir déclaré que Gobindolal se trouvait dans une impasse, de proposer cependant une autre fin: Bhromor aurait pardonné, si «Gobindolal s'était séparé à l'amiable de Rohini et s'était présenté les mains jointes devant [elle] ${ }^{24} \gg$.

Le roman toutefois ne narre pas cela. Bankim peut bien incriminer l'orgueil, la honte et la peur qui empêchent son protagoniste de se présenter devant Bhromor, il n'en demeure pas moins que le mariage n'a pas été pour Gobindolal une garantie contre l'égarement et que la disposition finale de Krishnokanto, qui visait probablement à ramener Gobindolal auprès de son épouse, n'a pas eu l'effet escompté. La référence à la mythologie citée plus haut est, de ce point de vue, porteuse d'une signification seconde qui consiste à s'invalider, ouvrant du même mouvement la voie au roman. Si le dieu Shiva put avaler le poison de Vasuki pour sauver les mondes de la destruction, gardant de l'épisode le nom de Nilakantha, «à la gorge bleue », il ne pouvait en aller de même pour Gobindolal, simple mortel. Dans l'imaginaire hindou, les hommes se repèrent à leur ombre que les dieux n'ont pas ${ }^{25}$ - ce qui vient quelque peu perturber l'image de la femme, ombre de Dieu. Laissant les dieux, la forme romanesque est celle qui parle des hommes, de leur ombre, de cette soif de beauté ${ }^{26}$ qui les altère et les livre à l'économie de la jouissance dont le testament n'est finalement que le signe ${ }^{27}$. Bankim se trouve par là pris, comme son personnage qui n'est pas sans caractère autobiographique, dans une tension sans solution : l'aspiration à l'harmonie est cela même qui le conduit à se cliver, à s'inscrire dans le romanesque comme son personnage $\mathrm{s}^{\prime}$ inscrit dans un destin ${ }^{28}$. Il se retrouve ainsi moderne par réticence à l'être.

23. Ibid., p. 193.

24. Ibid.

25. Voir par exemple l'épisode de Nala et Damayanti dans le Mahâbhârata, commenté par Charles Malamoud dans Cuire le monde. Rite et pensée dans l'Inde ancienne, Paris, La Découverte, 1989, p. 241-242.

26. Voir op. cit., p. 100.

27. Les rêves de Krishnokanto sous l'emprise de l'opium sont de ce point de vue exemplaires. Dans le premier (p. 45-46), le vieil homme attribue à des personnages mythologiques des actes économiques (hypothèque, paiement d'échéance); dans le second (p. 83-84), il imagine Rohini en train de voler le taureau de Shiva...

28. Bankim a repris à John Stuart Mill l'opposition entre la fatalité antique, grecque ou 
Le personnage de Rohini est exemplaire de cette tension, qui se traduit par divers amendements dans les éditions successives. La première édition montre une jeune femme impudique et cupide, qui disparaît des éditions suivantes ${ }^{29}$. Dans la quatrième et définitive, le narrateur semble hésiter; il la présente tantôt comme une femme malheureuse, prise de scrupule, souhaitant mourir dans sa détresse, et à laquelle il est malgré tout possible de montrer de la compassion :

Rohini continua de pleurer et je ne peux pas dire ce qui se passait dans son esprit. Peut-être se demandait-elle : pour quelle faute le sort m'a-t-il faite veuve si jeune? [...] Ceux qui peuvent jouir de tous les biens de la vie, comme la femme de Gobindolal, en quoi sont-ils plus vertueux que moi? $[\ldots]$

Nous avons déjà dit que Rohini n'était pas particulièrement bonne, et vous la voyez ici jalouse sans raison. Avec tant de défauts, comment pourrait-elle nous émouvoir par ses larmes? Elle ne nous émeut pas. Mais pourquoi cet éternel besoin de juger! Il est bien de pleurer à la seule vue des larmes des autres : les nuages des dieux n'arrêtent pas de pleuvoir à la vue d'un champ épineux.

Ayez donc un élan de compassion pour Rohini ${ }^{30}$.

Tantôt elle apparaît comme un « démon », une " pécheresse dépravée ${ }^{31}$ » qui ne peut résister au plaisir de la séduction, et à la dépravation de laquelle le roman ne saurait s'attacher: "On souhaiterait ici tirer le rideau pour éviter de montrer tout ce qui est impur et inconvenant. Mais nous ne raconterons que ce qu'il est indispensable de connaître ${ }^{32}$ », déclare le narrateur lorsqu'il évoque, dans la seconde partie du roman, la vie commune de Gobindolal et de Rohini. La moralité variable de la jeune femme apparaît comme le reflet des contradictions de l'artiste dans sa propre soif de beauté. Échappée à la sujétion et devenue sujet, inassignable, ne ressemblant ni à une fille légère ni à une veuve ${ }^{33}$, Rohini incarne l'individualité et ce n'est sans doute pas un hasard si Bankim, dans la présentation qu'il fait d'elle, use, en les niant, certes, de termes économiques :

La description de la beauté n'a pas cours sur le marché. Quant à la description de la vertu, les lois actuelles l'autorisent seulement pour

asiatique, et un « fatalisme modifié », ou destinée, résultant de lois physiques et psychologiques; voir le rapprochement à la page 119 entre la déchéance morale et la chute des corps, et Sisir Kumar Das, op. cit., p. 209.

29. Voir la préface de Nandadulal Dé, op. cit., p. 24.

30. KU, p. 58.

31. Ibid., p. 116 et 167.

32. Ibid., p. 156.

33. «Ce n'était pas son habitude d'aller en groupe avec des filles légères, au sourire léger tout au long du chemin et une cruche légère à la hanche. Sa cruche, à elle, ainsi que sa démarche, paraissait imposante et l'on reconnaissait bien là la veuve Rohini, quoiqu'elle ne ressemblât en rien d'autre à une veuve [...]»,op. cit., p. 54 . 
soi-même, non pour les autres. Disons donc simplement qu'elle était à la fleur de sa jeunesse, sa beauté débordait comme une lune d'automne dans son plein épanouissement ${ }^{34}$.

Comme la tradition, les anciens procédés d'écriture sont devenus obsolètes, ou ne peuvent subsister qu'accolés aux valeurs nouvelles. L'image du débordement qui est attachée à Rohini l'inscrit dans le monde nouveau, où la jouissance prévaut, irrésistible et mortifère.

Bankim écrivait des apologues pour illustrer sa dénonciation du sort des paysans. Il écrivit aussi de petits textes satiriques (Bransonism en 1882, Muchiramgurer Jîbancharit en 1884) mettant en scène les aliénations et les aberrations de l'Inde coloniale. On peut en avoir une idée avec la scène de corruption du facteur et l'enquête judiciaire dans KRSNakânter Uil. Mais un personnage tel que Rohini résiste à la forme de l'apologue, et il en va de même pour Gobindolal : l'un et l'autre sont représentés comme le terrain d'un combat entre Soumoti, «bonne pensée » et Koumoti, «mauvaise pensée ${ }^{35} »$. Le narrateur a beau se montrer sévère avec eux, il ne ruine pas complètement la sympathie du lecteur pour ces coupables divisés et meurtris. Le roman de la même façon ne peut se refermer sur lui-même, il demeure insatisfaisant, incomplet, il est travaillé par la tentation de l'idéalisation et de la simplification, mais celle-ci joue bien comme tentation, et l'allégorie du bien et du mal en vient à figurer la division du sujet ${ }^{36}$. Bankim disait fort justement du roman qu'il permettait l'exposition des problèmes complexes de la vie ${ }^{37}$. Genre apporté par le colonisateur, le roman devient ainsi non sans paradoxe, par son défaut, le genre de l'Inde moderne et de sa classe moyenne occidentalisée qui, à la différence de la classe moyenne européenne, n'est pas une classe de marchands mais de professions libérales et d'intellectuels. Avec le roman tel que Bankim le réinvente, ces Indiens d'un nouveau type acquièrent un mode d'expression à leur image, en quête d'une forme, mais de telle façon que la quête plus que la forme le définit. Dans KRSNakânter Uil où il n'est pas indifférent que Gobindolal soit montré lisant un roman lorsqu'il vit avec Rohini, alors que Bhromor pour dissiper son chagrin se plonge en vain dans l'Annadâmangal, célèbre poème de la littérature classique bengalie ${ }^{38}$, le chant du coucou (kokil), topos de la littérature sanskrite associé à l'érotisme printanier et aux scènes de réconciliation des amants, devient le signe d'une nostalgie existentielle qui préside à la rencontre de Rohini et de Gobindolal :

34. KU, p. 41.

35. Ibid., p. 61-62 et 130-132.

36. Les expérimentations narratives de Bankim peuvent aussi être analysées en ce sens : narration à la première personne dans la nouvelle Indirâ (1873); récit à quatre voix dans Rajanî, sur le modèle de The Woman on White de Wilkie Collins.

37. Voir Sisir Kumar Das, p. 65.

38. KU, p. 156 et 99 respectivement. 
[...] il semble, en l'entendant, que l'on ait perdu un être cher et que, par cette perte, toute la vie soit devenue vide, comme si l'on devait ne jamais le revoir. Il semble, sans que l'on sache pourquoi, qu'il nous manque quelque chose, une chose qui n'est pas réalisée et que l'on ne retrouvera plus. Il semble que l'on ait perdu un bijou quelque part, que quelqu'un appelle pour venir pleurer. Il semble que cette vie soit passée en vain, que la limite du bonheur n'ait pas été atteinte, que l'on n'ait rien goûté de l'éternelle beauté de cette terre ${ }^{39}$.

Or, le chapitre sixième qui comporte ce passage s'ouvre par la remarque suivante de l'auteur :

Ô kokil du printemps! Chante à cœur joie, je ne m’y oppose point; mais je te supplie de ne chanter qu'à l'heure propice. [...] Tu vois, après une longue recherche, j'ai trouvé une plume et un encrier, après une plus longue recherche encore, j'ai trouvé un sujet et, comme j'avais imaginé l'histoire du testament, j'allais me mettre à écrire, quand, du ciel, tu lanças ton kouhou.

Le chant du coucou perturbe donc l'auteur dans l'écriture de son histoire qui, sous l'effet d'une aspiration diffuse, recommence en ce début de sixième chapitre : «Que je vous raconte d'abord ce qui s'est passé quand [Rohini] alla chercher de l'eau. Voici l'histoire [...]. » Mais cette aspiration s'entend aussi comme inspiration, où l'on reconnaît les accents du romantisme européen, ceux par exemple de « To the Cuckoo » de Wordsworth ${ }^{40}$. Bankim toutefois n'écrit pas de poésie, il y a renoncé après quelques poèmes de jeunesse peu convaincants ${ }^{41}$ : sa prose romanesque ne s'inspire pas directement de la littérature anglaise, elle présente avec elle des affinités au terme d'un parcours complexe où l'écrivain invente sa propre modernité, empruntant un genre, le roman, y intégrant des procédés de la poésie sanskrite, les chargeant d'un sens nouveau qui résulte de l'impossibilité désormais de coïncider avec soi-même. La modernité européenne joue donc chez Bankim à plusieurs niveaux qui entrent en tension pour constituer une modernité indienne, ce qu'un critique indien exprime en déclarant: « His novels are born out of the conflict of two apparently contradictory ideals, romanticism on the one hand and the doctrine of utilitarianism on the other ${ }^{42}$. » $\mathrm{La}$ rupture, quoi qu'il en soit, est donc aussi celle de la modernité européenne elle-même, travaillée de l'intérieur, par l'extérieur.

À bien des égards, l'œuvre de Bankim est une œuvre sur les poisons. $\mathrm{Au}$ « poison de la discrimination » dénoncé dans l'essai s'ajoute et s'oppose

39. KU, p. 55.

40. «Thrice welcome, darling of the Spring! / Even yet thou art to me / No bird, but an invisible thing, / A voice, a mystery; // The same whom in my school-boy days / I listened to; that Cry / Which made me look a thousand ways / In bush, and tree, and sky. », dans W. Wordsworth, The Major Works including The Prelude, Oxford World's Classics, 2000, p. 245 .

41. « His verse is dull and wooden », Sisir Kumar Das, op. cit., p. 8.

42. Sisir Kumar Das, op. cit., p. 208. 
le poison que met en scène le roman, celui de la passion, du désir infini, de l'aspiration à la beauté. Entre les deux se situe le remède empoisonné, le pharmakon qu'est Rousseau, antidote au premier poison, mais de la même nature séductrice que le second. La modernité apparaît alors elle-même comme un pharmakon, délivrant d'un mal en en libérant un autre. Double, elle ne requiert pas moins de deux formes de discours, et les deux genres pratiqués par Bankim, l'essai et le roman, peuvent être analysés dans cette perspective comme complémentaires, l'essai interrogeant l'articulation du poison social et de son remède quand le roman s'attelle au remède et à sa vénénosité. La dichotomie de Bankim se comprend dès lors moins comme une duplicité, un «Jekyll-and-Hyde framework » comme le formule Sisir Kumar Das pour le contester ${ }^{43}$, que le double visage de Janus bifrons, le dieu romain des ouvertures et des passages, temporels et spatiaux. De la même façon, l'œuvre de Bankim est celle d'une subjectivité, moderne au sens propre si l'on se souvient que le mot, formé sur modo, « juste », « justement », ne prend son sens temporel que dans une situation d'énonciation précise $^{44}$. Parce que l'individu est double, il ne peut y avoir chez Bankim opposition simple entre l'Inde et l'Europe, l'ancien et le nouveau, le roman et l'essai. C'est bien plutôt le chiffre trois qui semble organiser sa personnalité et son œuvre : trois langues, trois littératures, trois poisons. A partir des années 1880, Bankim, il est vrai, aura la tentation d'intégrer l'abolition de l'opposition entre l'ancien et le nouveau dans un hindouisme englobant dont on trouve une préfiguration dans Sâmya lorsque la Révolution française est incidemment comparée à un " sacrifice ${ }^{45}$ ». Mais cela même ne peut être simple, et l'étrange roman d'Ânandamath / Le Monastère de la félicité, en 1884, ne manque généralement pas de surprendre le lecteur par sa fin où, bien qu'ayant triomphé des soldats musulmans au service des Anglais, les renonçants hindous qui ont pris les armes pour libérer leur Mère l'Inde sont invités à accepter, pour un temps du moins, l'avènement d'un royaume britannique. Le roman fuit sa clôture : du protagoniste de KRSNakânter Uil on retient moins les répliques finales de l'ascète (« Le seul moyen de trouver la paix est d'offrir son âme à Dieu. Maintenant c'est lui qui est ma propriété, c'est Lui qui est ma Bhromor $\left.{ }^{46} \gg\right)$ que les silences et les absences d'un jeune homme à qui il fut impossible d'être un héritier.

Claudine Le Blanc

Université Paris 3 - Sorbonne Nouvelle

43. Sisir Kumar Das, op. cit., "Introduction », p. X.

44. Dans des énoncés comme «il arrive juste», «il vient juste de partir», voir H. Meschonnic, op. cit., p. 36-37, note 27.

45. S, p. 162.

46. KU, p. 200. On notera que dans les trois premières versions du roman, Gobindolal se suicidait. 